\title{
Plasma exchange in severe acute relapses of multiple sclerosis - Results from a Portuguese cohort
}

\author{
Inês Correia ${ }^{\mathrm{a}, *}$, Joana Jesus Ribeiro ${ }^{\mathrm{a}}$, Luís Isidoro ${ }^{\mathrm{b}}$, Sónia Batista ${ }^{\mathrm{a}}$, Carla Nunes ${ }^{\mathrm{a}}$, Carmo Macário ${ }^{\mathrm{a}}$, \\ Catarina Borges ${ }^{\mathrm{c}}$, Jorge Tomaz ${ }^{\mathrm{c}}$, Lívia Sousa ${ }^{\mathrm{a}}$ \\ a Department of Neurology, Centro Hospitalar e Universitário de Coimbra, Coimbra, Portugal \\ b Department of Neurology, Hospital de São Teotónio, Viseu, Portugal \\ ${ }^{\mathrm{c}}$ Blood and Transfusion Medicine department, Centro Hospitalar e Universitário de Coimbra, Coimbra, Portugal
}

\section{A R T I C L E I N F O}

\section{Keywords:}

Multiple Sclerosis

Relapse

Plasma exchange

Treatment

\begin{abstract}
A B S T R A C T
Background: Relapses in Multiple Sclerosis (MS) are often associated with significant disability impairment which is resultant from poor response to corticosteroids. In such severe cases, plasma exchange (PLEX) may be used, although only a few studies with MS patients have been reported. Our objective was to evaluate the effectiveness of PLEX in severe relapses of MS.

Methods: Retrospective study of MS patients treated with PLEX in acute relapses. Data regarding EDSS, annualized relapse rate (ARR), treatment with corticosteroids, number of PLEX sessions, adverse events, and gadolinium enhancement in brain MRI were analysed.

Results: Included 46 patients, $76.09 \%$ female $(\mathrm{n}=35)$ with mean age of 38.76 years and mean disease duration of 5.99 years, of which $84.78 \%$ had a Relapsing Remitting MS ( $n=39$ ), 15.22\% Secondary Progressive MS ( $n=$ 7). The previous ARR was 1.1 and in $28.26 \%$ of the cases $(n=13)$ PLEX was used in the relapse that led to MS diagnosis. The majority of relapses had motor impairment $(69.6 \%, n=32)$, with a median EDSS increase of 1.5 points from baseline (maximum of 6.5 ) and higher than 1.5 points in $45.65 \%$ of cases $(n=21)$. Brain MRI was available in $69.57 \%$ of the cases $(n=32)$, and gadolinium enhancing lesions were present in $68.75 \%$ of cases $(n$ $=22$ ). Corticosteroids were used before PLEX in all patients for a mean of 6.09 days, without any immediate benefit in $41.30 \%$ of cases $(n=19)$, with the remaining cases showing only mild disability recovery. After a mean of 7.39 PLEX sessions, there was clinical benefit with complete EDSS recovery in $41.30 \%$ of patients ( $\mathrm{n}=$ 19), and partial in $39.13 \%(n=18)$. There were no adverse events related to PLEX in $89.13 \%$ of patients ( $n=$ 41) and in the remaining patients the reported adverse events included deep venous thrombosis $(n=1)$, anaemia $(n=1)$, fever $(n=1)$, hypoalbuminemia $(n=1)$ and arterial hypotension $(n=1)$.

Conclusion: Our results support the use of PLEX in severe relapses unresponsive to corticosteroids, since it was an effective and relatively safe treatment for most of our patients.
\end{abstract}

\section{Introduction}

Multiple Sclerosis (MS) is the most common demyelinating inflammatory disease of the central nervous system (CNS), and the leading cause of non-traumatic neurological disability in young adults in North America and Europe, affecting more than two million people worldwide (Dutta and Trapp 2011).

Most patients experience a relapsing remitting MS (RRMS), with acute episodes of neurologic dysfunction named relapses, followed by periods of partial or complete remission with clinical stability between episodes, although patients with secondary progressive MS (SPMS) and primary progressive MS (PPMS) may also experience relapses during evolution of the disease (Confavreux et al., 2000; Lublin et al., 2014).

Although in the past 20 years an important progress has been made in the field of multiple sclerosis treatment, the available treatments are yet incapable of completely preventing relapse occurrence, and its treatment is still a major component of the management of MS patients, in all phases of the disease (Comi et al., 2017). Relapses with incomplete remissions are an important contributor for the increase in neurologic disability and decreased health-related quality of life, and therefore aggressive relapse treatment is critical (Confavreux et al., 2000; Berkovich, 2013).

The gold standard treatment for MS relapses are corticosteroids. Several mechanisms have been proposed to justify its efficacy: decrease

\footnotetext{
* Correspondence to: Av. Bissaya Barreto - Praceta Prof. Mota Pinto, 3000-075 Coimbra, Portugal

E-mail address: mcorreia.ines@gmail.com (I. Correia).
} 
of oedema due to anti-inflammatory component; reduction of B-lymphocyte counts and their availability at the inflammatory lesions resulting in a decreased number of immunoglobulin (Ig) G synthesizing cells in the CNS; reduction of the blood-brain barrier abnormally increased permeability resulting in less gadolinium enhancing lesions (Berkovich, 2013; Frohman et al., 2007; Durelli et al., 1986; Martinelli et al., 2009).

Frequently, severe relapses are unresponsive to corticosteroids and in such cases, several options have been studied and used, such as plasma exchange (PLEX), cyclophosphamide, intravenous immunoglobulin G (IVIG) and natalizumab (Berkovich, 2013).

Several reports of PLEX efficacy have been published and although most studies have small cohorts and different evaluation methods, (Dau et al., 1980; Valbonesi et al., 1981; Weiner et al., 1989; Palm et al., 1991; Weinshenker et al., 1999; Weinshenker, 1999; Keegan et al., 2002, 2005; Bennetto et al., 2004; Ruprecht et al., 2004; Schilling et al., 2006; Linker et al., 2007; Llufriu et al., 2009; Trebst et al., 2009; Habek et al., 2010; Roesner et al., 2012; Ehler et al., 2015; Ikeda et al., 2015; Deschamps et al., 2016, 2017) due to the available evidence, European and American guidelines consider that some patients with MS, who have not responded to treatment with methylprednisolone may benefit from PLEX (Sellebjerg et al., 2005; Cortese et al., 2011).

Our objective was to evaluate efficacy and safety of PLEX in a cohort with severe relapses of MS refractory to corticosteroids treatment, in order to reinforce its importance in the management of MS relapses.

\section{Materials and methods}

\subsection{Participants}

This was a retrospective study. The start date for inclusion was January 1, 2000 and the last date for data introduction was 31 December 2015. The inclusion criteria were: patients followed in the Neurology Department of our Portuguese University Hospital with the diagnosis of RRMS (all patients met the criteria for diagnosis according to the McDonald Criteria of 2010); (Polman et al., 2011) treatment with Plasma Exchange (PLEX) during an acute relapse; and age above 18 years. Exclusion criteria were incomplete medical records.

This study was approved by the local ethics committee.

\subsection{Clinical assessment}

A relapse was defined as current patient-reported symptoms or objectively observed signs, typical of an acute inflammatory demyelinating event in the central nervous system, lasting at least $24 \mathrm{~h}$, in the absence of fever or infection (Polman et al., 2011).

Data regarding MS subtype, EDSS, annualized relapse rate (ARR), previous and following treatment, treatment with corticosteroids, number of PLEX sessions, adverse events, and gadolinium enhancement in brain MRI were analysed.

\subsection{MRI acquisition and analysis}

Data from 1.5 T MRI reports were obtained. The presence of gadolinium enhancement lesions in brain MRI was analysed.

\subsection{Statistical analysis}

Demographic characteristics were presented as means and standard deviations for continuous variables, and as frequencies and percentages for categorical variables. The ARR and the EDSS were described as the mean and standard deviation. Safety outcomes were reported descriptively. In our sample all the variables have a non-normal distribution, so nonparametric tests were used. The Wilcoxon Signed Ranks Test was used to compare related continuous and related ordinal samples, and the Mann-Whitney Test is used to compare different groups of patients.
Table 1

Patient characteristics previous to PLEX treatment.

\begin{tabular}{ll}
\hline Female gender, \% (n) & $76.09 \%(\mathrm{n}=35)$ \\
Mean age, years (min-max, SD) & $38.76(19-71$, \\
& $10.30)$ \\
Mean disease duration, years (min-max, SD) & $5.99(0-19,6.0)$ \\
Subtype MS & \\
- RRMS, \% (n) & $84.78 \%(\mathrm{n}=39)$ \\
- SPMS, \% (n) & $15.22 \%(\mathrm{n}=7)$ \\
Annual relapse rate in the previous year, mean (min-max, & $1.13(0-4,1.11)$ \\
$\quad$ SD) & \\
Baseline EDSS, median (min-max, IQR) & $2.49(0-6.5,2.9)$ \\
Baseline MS treatment & \\
- Naïve - without previous MS diagnosis \% (n) & $28.26 \%(\mathrm{n}=13)$ \\
- Glatiramer acetate, \% (n) & $13.04(\mathrm{n}=6)$ \\
- Interferon beta, \% (n) & $34.78(\mathrm{n}=16)$ \\
- Fingolimod, \% (n) & $8.70(\mathrm{n}=4)$ \\
- Natalizumab, \% (n) & $2.17(\mathrm{n}=1)$ \\
- Azathioprine, \% (n) & $4.35(\mathrm{n}=2)$ \\
- Micophenolate mofetil & $2.17(\mathrm{n}=1)$ \\
- Mitoxantrone & $2.17(\mathrm{n}=1)$ \\
- Cyclophosphamide & $4.35(\mathrm{n}=2)$ \\
\hline
\end{tabular}

Abbreviations: $\mathrm{n}$ - number; MS- Multiple Sclerosis; RRMS - relapsing-remitting multiple sclerosis; min - minimum; max - maximum; SD - standard deviation; IQR - interquartile range.

$\mathrm{P}$ values (2-tailed) $<0.05$ were considered statistically significant; in the graphs $95 \%$ confidence intervals were used.

\section{Results}

\subsection{Demographic characteristics of the patients}

Among the MS population regularly followed in our outpatient department ( $\mathrm{n}=1014$ patients), 47 patients were treated with PLEX, of which one was excluded due to incomplete medical records, with a final population of 46 patients. Patient baseline characteristics are summarized in Table 1.

The diagnosis of MS was performed in the relapse leading to PLEX treatment in $28.26 \%$ of the cases ( $n=13)$. In $23.91 \%$ of the cases ( $n=$ 11) patients were treated with fingolimod, natalizumab or other immunosuppressive therapies. In the particular case of other immunosuppressive therapies, azathioprine was used in two patients (RRMS and SPMS) in 2007, micophenolate mofetil was used in one RRMS patient in 2014, mitoxantrone was used in one RRMS patient in 2009, cyclophosphamide was used in one RRMS patient in 2007 and in another with SPMS in 2014. These treatments were used in all cases after approved disease modifying treatments failure.

\subsection{PLEX in our cohort}

\subsubsection{Relapse characteristics}

The relapse characteristics that led to PLEX may be consulted in Table 2.

Table 2

Relapse characteristics that led to PLEX.

\begin{tabular}{ll} 
Symptoms & \\
- Motor impairment, \% (n) & $47.83 \%(\mathrm{n}=22)$ \\
- Severe optic neuritis, \% (n) & $4.35 \%(\mathrm{n}=2)$ \\
- Ataxia, \% (n) & $4.35 \%(\mathrm{n}=2)$ \\
- Brainstem symptoms, \% (n) & $4.35 \%(\mathrm{n}=2)$ \\
- Multifocal symptoms, \% (n) & $39.13 \%(\mathrm{n}=18)$ \\
EDSS during relapse, median (min-max, IQR) & $4.0(1.5-8.0,3.0)$ \\
EDSS increase during relapse, median (min-max, IQR) & $1.5(0-6.5,2.0)$ \\
Gadolinium enhancing lesions in brain $\mathrm{MRI}^{\mathrm{a}}, \%(\mathrm{n})$ & $68.8 \%(\mathrm{n}=22)$ \\
\hline
\end{tabular}

Abbreviations: PLEX - plasma exchange; $\mathrm{n}$ - number; min - minimum; max - maximum; IQR - interquartile range; MRI - magnetic resonance imaging.

a - MRI available in 32 patients. 


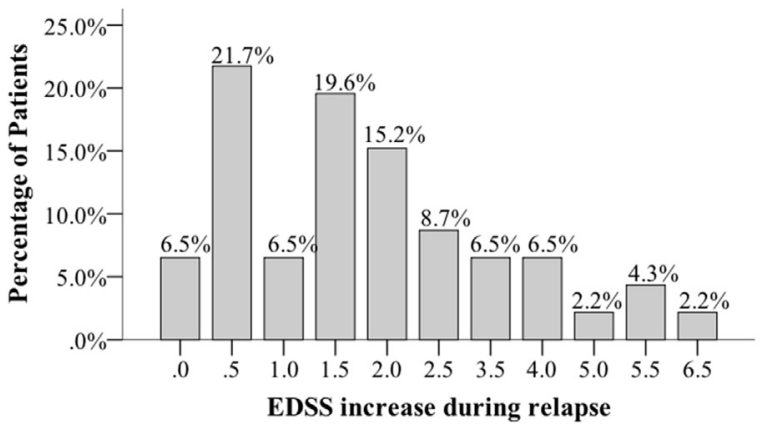

Fig. 1. Distribution of patients according to the disability increase measured by EDSS points during relapse.

The majority of the cases experienced severe disability, with median EDSS during relapse of 4.0, after a median increase of 1.5 points from baseline (maximum of 6.5). In fact, the EDSS increased over 1.5 points in $45.65 \%$ of the patients $(n=21)$, as reported in Fig. 1 .

In $6.52 \%$ of the patients $(n=3)$ the impairment provoked by the relapse did not lead to an increase in EDSS. One of these cases was a patient with baseline EDSS of 3.0 who experienced a severe optic neuritis without EDSS increase. The other two patients had a baseline EDSS of 3.5 and 3.0 respectively, and experienced a worsening of previous paraparesis without measured EDSS increase.

In the particular cases of previously undiagnosed patients, the median EDSS during relapse was 3.5.

Brain MRI performed during the relapse was available in $69.57 \%$ of the cases $(n=32)$. In those patients, gadolinium enhancing lesions were present in $68.75 \%$ of cases $(n=22)$.

\subsubsection{Relapse treatment before PLEX}

All patients were previously treated with corticosteroids (methylprednisolone $1 \mathrm{~g}$ administered intravenously once daily) before starting PLEX.

The mean duration of corticosteroids treatment was 6.09 days and treatment duration with methylprednisolone may be consulted in Table 3.

Despite the treatment with corticosteroids, $41.30 \%$ of the patients ( $n=19$ ) did not experienced any immediate benefit, and the remaining cases had showed only mild disability recovery. In the subgroup of patients without MS previously diagnosed, 30.77\% (n = 4) did not improve with corticosteroids, and the remaining only experienced partial recovery.

\subsubsection{PLEX treatment}

PLEX was started after corticosteroids treatment in all patients, in mean, 33.48 days after relapse onset (Table 4).

The distribution of patients according to the number of PLEX sessions may be consulted in Table 4. One patient only performed one session stopped the treatment due to the loss of the intravenous access.

The median EDSS after PLEX was 2.75 (minimum of 1.0 and maximum of 8.0, IQR 2.1).

Our population experienced recovery of neurological disability in $80.43 \%$ of the cases $(n=37)$ (Table 4$)$. Considering the subgroup of patients without MS previously diagnosed $(n=13)$, none of the

Table 3

Methylprednisolone treatment before PLEX.

\begin{tabular}{ll}
\hline Mean treatment duration, days (SD) & $6.09(1.94)$ \\
$3-4$ days, n (\%) & $3(6.52)$ \\
5 days, n (\%) & $26(56.52)$ \\
7 days, n (\%) & $10(21.74)$ \\
2 cycles of treatment (5 days each), n (\%) & $7(15.22)$ \\
\hline
\end{tabular}

Abbreviations: PLEX - plasma exchange; SD - standard deviation; $\mathrm{n}$ - number.
Table 4

Treatment with PLEX.

$\begin{array}{ll}\text { Treatment initiation since relapse onset } & \\ \text { - Mean duration, days (min-max, SD) } & 33.48(10-120,24.22) \\ \text { - 2nd week, n (\%) } & 9(19.57) \\ \text { - 3rd-4th week, n (\%) } & 9(19.57) \\ \text { - 5th- 6th week, n (\%) } & 20(43.48) \\ \text { - 3rd month, n (\%) } & 5(10.87) \\ \text { - 4th month, n (\%) } & 2(4.35) \\ \text { - 5th month, n (\%) } & 1(2.17) \\ \text { Number of PLEX sessions (every other day) } & \\ \text { - Mean (min-max, SD) } & 7.39(1-14,2.79) \\ \text { - } 1-6, \mathrm{n}(\%) & 17(36.96) \\ \text { - } 7-10, \mathrm{n}(\%) & 26(56.52) \\ \text { - } 11-14, \mathrm{n}(\%) & 3(6.52) \\ \text { Disability recovery after PLEX } & \\ \text { - Complete, n (\%) } & 19(41.3) \\ \text { - Partial, n (\%) } & 18(39.13) \\ \text { - None, n (\%) } & 9(19.56)\end{array}$

Abbreviations: PLEX - plasma exchange; min - minimum; max - maximum; SD - standard deviation; $\mathrm{n}$ - number; \% - percentage.

patients completely recovered immediately after PLEX, but partial recovery was achieved in $69.23 \%$ of the cases $(n=9)$.

Considering the different groups of response to PLEX, there was a significant difference in the number of PLEX sessions between groups ( $\mathrm{p}$ $=0.010$ ). The subgroup of patients who experienced complete disability recovery had the highest number of PLEX sessions (mean of 9.26 sessions), compared to those patients with partial recovery (mean of 7.17 sessions), and the number was even lower in those patients who did not recover (mean of 5.78 sessions).

There were no other significant differences between groups, neither regarding gender, disease duration, previous disease modifying treatment, type of relapse or the presence of gadolinium enhancing lesions on brain MRI. Regarding time to PLEX since relapse onset we found no significant differences between groups, with disability recovery (partial or complete) occurring in $83.33 \%$ of the patients who performed PLEX during the first month after relapse onset ( $n=30$ of 36 patients), $60 \%$ in the patients who performed PLEX during the second month after relapse onset ( $\mathrm{n}=3$ of 5 patients), $75 \%$ during the third month ( $\mathrm{n}=3$ of 4 patients), and the patient who had PLEX after 4 months since relapse onset experienced complete recovery.

Late response to PLEX was evaluated considering EDSS assessed one year later, with improvement in EDSS of $10.87 \%$ of the patients $(n=5)$ and stability in $80.44 \%$ of the patients $(n=37)$. Overall, although a decrease in median EDSS was observed (2.5, minimum of 1.0 and maximum of 8.0, IQR 2.5), it was not significantly different from the EDSS evaluated immediately after PLEX.

After PLEX treatment, $47.82 \%(n=22)$ of the patients who were already under MS treatment $(\mathrm{n}=33$ ) changed the disease modifying treatment. Considering the entire population, after this relapse $45.65 \%$ $(n=21)$ of the patients were treated with natalizumab or fingolimod, and $21.7 \%(\mathrm{n}=10)$ were treated with immunosuppressive drugs such as cyclophosphamide $(n=4)$, rituximab $(n=3)$, and azathioprine ( $=3$ ).

\subsubsection{PLEX related adverse events}

PLEX was a safe treatment for most of the patients since there were no adverse events reported in $89.13 \%$ of the patients $(n=41)$.

In the remaining patients, a few adverse events were reported. One patient experienced deep venous thrombosis after 14 sessions, leading to PLEX suspension. It was not necessary to stop PLEX in the other patients with side effects, which were: mild anaemia $(n=1)$, transient fever $(n=1)$, hypoalbuminemia corrected with intravenous reposition of albumin without PLEX discontinuation $(\mathrm{n}=1)$ and arterial hypotension without complications or PLEX discontinuation $(\mathrm{n}=1)$. 


\section{Discussion}

The majority of the published studies have in common the inclusion of patients with MS experiencing with aggressive diseases and severe relapses (Weiner et al., 1989; Weinshenker, 1999). The demographic characteristics of our population were similar to those described in most published studies, with a greater proportion of RRMS subtype (84.8\%) and female gender (76.09\%). However, these patients had also a very aggressive disease, with high mean relapse rate in the previous year (1.1) and accumulated disability with median EDSS of 2.25. Also, $23.91 \%$ of the patients were already treated with immunosuppressive drugs. The high increase in median EDSS during relapse was related to the type of symptoms experienced by the patients (mainly motor), since EDSS highly relies on motor function disability. The majority of cases with available brain MRI showed gadolinium enhancing lesions (68.75\%), and if spinal MRI was available this number would probably be even higher. Even considering the patients without MS previously diagnosed, the relapse that led to PLEX was very severe (median EDSS during relapse of 3.5), and patients did not have an adequately response to corticosteroids. In all the cases, PLEX was used as a second-line treatment after corticosteroids, in order to improve recovery and avoid residual deficits.

The mean number of PLEX sessions in our population (7.39 sessions every other day) was comparable to other studies previously described (Weinshenker, 1999; Keegan et al., 2002; Llufriu et al., 2009; Ehler et al., 2015). Although good results were achieved in one study with lower number of PLEX sessions (range 3-5) (Bennetto et al., 2004), in our population there was a significant difference towards greater recovery with higher number of PLEX sessions. These results endorse the need to adjust the number of sessions to the specific clinic situation in order to achieve a better income.

The majority of our patients improved after PLEX, with complete recover of neurological disability achieved in $41.30 \%$ of the cases and partial recovery in $39.13 \%$. This corroborate the results of previous studies, where recovery rates of ranging from $44.1 \%$ to $72.2 \%$ have been reported (Weinshenker, 1999; Keegan et al., 2002; Bennetto et al., 2004; Llufriu et al., 2009; Ehler et al., 2015).

We also found that the EDSS achieved with PLEX treatment appeared to be stable after one year. This has been previously reported in some studies (Weinshenker, 1999; Llufriu et al., 2009), but not all, (Keegan et al., 2005) and may have several explanations. We could speculate that PLEX is associated with a lasting immunomodulatory effect. However, it is reasonably to attribute part of this stability to a switch or an escalation in the disease modifying treatment after the severe relapse. In fact, $47.82 \%$ of our patients who were already under MS treatment switched or escalated treatment, and $45.65 \%$ of our population was treated with natalizumab or fingolimod after this relapse.

Some studies have highlighted some predictors of good response after PLEX such as male sex, retained reflexes (Keegan et al., 2002), gadolinium enhancing lesions in MRI (Ehler et al., 2015), early initiation of PLEX (Keegan et al., 2002; Llufriu et al., 2009). In our study we did not find a significant relation between these or other variables, as in another study (Keegan et al., 2005), except for the number of PLEX sessions as previously discussed. In fact, regarding time to PLEX since relapse onset, patients experienced disability recovery even when it was performed a long time after the relapse (83.33\% during the first month, $60 \%$ during the second month, $75 \%$ during the third month and even the patient who had PLEX after 4 months experienced complete recovery), as it was already previously described (Bennetto et al., 2004).

The mechanism of action of PLEX in MS has been explored. Some patients seem to respond promptly to PLEX, while other do not respond at all. To explain this dichotomy, some authors propose that biological factors such as MS immunopathology heterogeneity between patients could contribute to differences in PLEX response Keegan et al. (2005). Four immunopathological patterns of demyelination in early multiple sclerosis lesions have been described: in pattern I T-cell/macrophage- associated demyelination occurs; pattern II is characterised by immunoglobulin deposition and complement activation with antibody/ complement-associated demyelination; pattern III is defined by a distal oligodendrogliopathy; and pattern IV is characterised by oligodendrocyte degeneration in periplaque white matter (Lucchinetti et al., 2000). In one study who particularly investigated the response to PLEX according to immunopathological pattern, all patients with pattern II pathology experienced moderate-to-marked improvement, compared to none of the remaining patients with different patterns Keegan et al. (2005). This selective response suggests that the mechanism of action of PLEX relies on removal of immunoglobulins, complement factors, cytokines, and immune complexes, and probably not as much on T-cell and macrophage inflammation which is similar across all three immunopathological patterns (Keegan et al., 2005) In fact, the success of PLEX in other presumed humorally mediated disorders such as neuromyelitis optica support this assumption (Keegan et al., 2005).

A minority of our patients experienced some side effects related to PLEX, most of them easily manageable and not requiring PLEX discontinuation. This finding was comparable previous descriptions, in which the majority of adverse events were incidental or related to the patient's underlying illness (Weinshenker, 1999). Common adverse effects described include anaemia and symptomatic hypotension (Weinshenker, 1999; Keegan et al., 2002; Llufriu et al., 2009). Heparinassociated thrombocytopenia has been described in 2 patients (Keegan et al., 2002), bacteraemia due to infection of their central venous line was also described in 2 patients (concomitant vein thrombose in one patient) and a rash successfully resolved with oral corticosteroids was described in one patient (Llufriu et al., 2009). In one study, central IV catheter placement was necessary in $44.1 \%$ of the patients (Keegan et al., 2002).

The limitations of our study are mostly related to the retrospective nature of the data collection. Moreover, it represents a single-centred, observational and nonrandomized study, with a small population included and without of a control group.

In our study, we have contributed to establishing the effectiveness of PLEX regarding the recovery from severe relapses.

\section{Conclusion}

Our results support the use of PLEX in severe relapses unresponsive to corticosteroids. The treatment was effective and relatively safe for most of our patients. Considering our results and the available state of art, we agree it should considered in the case of a relapse without complete recovery after corticosteroids.

\section{Conflict of interests}

Dr. Inês Correia: nothing to disclose.

Dr. Joana Jesus-Ribeiro: nothing to disclose.

Dr. Luís Isidoro: nothing to disclose.

Dr. Sónia Batista has received grant support from Biogen and speakers' bureau fees from Biogen, Novartis and Merck.

Dr. Carla Nunes has received speakers' bureau fees and have been advisory board member for Biogen, Novartis, Teva, Bayer, Genzyme and Merck.

Dr. Carmo Macário has received speakers' bureau fees and have been advisory board member for Biogen, Novartis, Teva, Bayer, Genzyme and Merck.

Dr. Catarina Borges: nothing to disclose.

Dr. Jorge Tomaz: nothing to disclose.

Dr. Lívia Sousa has received speakers' bureau fees and have been advisory board member for Biogen, Novartis, Teva, Bayer, Genzyme and Merck. 


\section{References}

Bennetto, L., Totham, A., Healy, P., Massey, E., Scolding, N., 2004. Plasma exchange in episodes of severe inflammatory demyelination of the central nervous system. A report of six cases. J. Neurol. 251 (12), 1515-1521.

Berkovich, R., 2013. Treatment of acute relapses in multiple sclerosis. Neurotherapeutics 10 (1), 97-105.

Comi, G., Radaelli, M., Soelberg Sorensen, P., 2017. Evolving concepts in the treatment of relapsing multiple sclerosis. Lancet 389 (10076), 1347-1356.

Confavreux, C., Vukusic, S., Moreau, T., Adeleine, P., 2000. Relapses and progression of disability in multiple sclerosis. N. Engl. J. Med. 343 (20), 1430-1438.

Cortese, I., Chaudhry, V., So, Y.T., Cantor, F., Cornblath, D.R., Rae-Grant, A., 2011. Evidence-based guideline update: plasmapheresis in neurologic disorders: report of the therapeutics and technology assessment subcommittee of the American Academy of Neurology. Neurology 76 (3), 294-300.

Dau, P.C., Petajan, J.H., Johnson, K.P., Panitch, H.S., Bornstein, M.B., 1980. Plasmapheresis in multiple sclerosis: preliminary findings. Neurology 30 (10), 1023-1028.

Deschamps, R., Gueguen, A., Parquet, N., Saheb, S., Driss, F., Mesnil, M., et al., 2016. Plasma exchange response in 34 patients with severe optic neuritis. J. Neurol. 263 (5), 883-887.

Deschamps, R., Gueguen, A., Parquet, N., Saheb, S., Driss, F., Mesnil, M., et al., 2017. Erratum to: plasma exchange response in 34 patients with severe optic neuritis. J Neurol 264. pp. 1547.

Durelli, L., Cocito, D., Riccio, A., Barile, C., Bergamasco, B., Baggio, G.F., et al., 1986. High-dose intravenous methylprednisolone in the treatment of multiple sclerosis: clinical-immunologic correlations. Neurology 36 (2), 238-243.

Dutta, R., Trapp, B.D., 2011. Mechanisms of neuronal dysfunction and degeneration in multiple sclerosis. Prog. Neurobiol. 93 (1), 1-12.

Ehler, J., Koball, S., Sauer, M., Mitzner, S., Hickstein, H., Benecke, R., et al., 2015. Response to therapeutic plasma exchange as a rescue treatment in clinically isolated syndromes and acute worsening of multiple sclerosis: a retrospective analysis of 90 patients. PLoS One 10 (8), e0134583.

Frohman, E.M., Shah, A., Eggenberger, E., Metz, L., Zivadinov, R., Stuve, O., 2007. Corticosteroids for multiple sclerosis: I. Application for treating exacerbations. Neurotherapeutics 4 (4), 618-626.

Habek, M., Barun, B., Puretic, Z., Brinar, V.V., 2010. Treatment of steroid unresponsive relapse with plasma exchange in aggressive multiple sclerosis. Ther. Apher. Dial. 14 (3), 298-302.

Ikeda, K.M., Lee, D.H., Fraser, J.A., Mirsattari, S., Morrow, S.A., 2015. Plasma exchange in a patient with tumefactive, corticosteroid-resistant multiple sclerosis. Int. J. MS Care. 17 (5), 231-235.

Keegan, M., Pineda, A.A., McClelland, R.L., Darby, C.H., Rodriguez, M., Weinshenker, B.G., 2002. Plasma exchange for severe attacks of CNS demyelination: predictors of response. Neurology 58 (1), 143-146.

Keegan, M., Konig, F., McClelland, R., Bruck, W., Morales, Y., Bitsch, A., et al., 2005. Relation between humoral pathological changes in multiple sclerosis and response to therapeutic plasma exchange. Lancet 366 (9485), 579-582.

Linker, R.A., Chan, A., Sommer, M., Koziolek, M., Muller, G.A., Paulus, W., et al., 2007.
Plasma exchange therapy for steroid-refractory superimposed relapses in secondary progressive multiple sclerosis. J. Neurol. 254 (9), 1288-1289.

Llufriu, S., Castillo, J., Blanco, Y., Ramio-Torrenta, L., Rio, J., Valles, M., et al., 2009. Plasma exchange for acute attacks of CNS demyelination: predictors of improvement at 6 months. Neurology 73 (12), 949-953.

Lublin, F.D., Reingold, S.C., Cohen, J.A., Cutter, G.R., Sorensen, P.S., Thompson, A.J., et al., 2014. Defining the clinical course of multiple sclerosis: the 2013 revisions. Neurology 83 (3), 278-286.

Lucchinetti, C., Bruck, W., Parisi, J., Scheithauer, B., Rodriguez, M., Lassmann, H., 2000. Heterogeneity of multiple sclerosis lesions: implications for the pathogenesis of demyelination. Ann. Neurol. 47 (6), 707-717.

Martinelli, V., Rocca, M.A., Annovazzi, P., Pulizzi, A., Rodegher, M., Martinelli Boneschi, F., et al., 2009. A short-term randomized MRI study of high-dose oral vs intravenous methylprednisolone in MS. Neurology 73 (22), 1842-1848.

Palm, M., Behm, E., Schmitt, E., Buddenhagen, F., Hitzschke, B., Kracht, M., et al., 1991. Immunoadsorption and plasma exchange in multiple sclerosis: complement and plasma protein behaviour. Biomater. Artif. Cells Immobil. Biotechnol. 19 (1), 283-296.

Polman, C.H., Reingold, S.C., Banwell, B., Clanet, M., Cohen, J.A., Filippi, M., et al., 2011. Diagnostic criteria for multiple sclerosis: 2010 revisions to the McDonald criteria. Ann. Neurol. 69 (2), 292-302.

Roesner, S., Appel, R., Gbadamosi, J., Martin, R., Heesen, C., 2012. Treatment of steroidunresponsive optic neuritis with plasma exchange. Acta Neurol. Scand. 126 (2), $103-108$.

Ruprecht, K., Klinker, E., Dintelmann, T., Rieckmann, P., Gold, R., 2004. Plasma exchange for severe optic neuritis: treatment of 10 patients. Neurology 63 (6), 1081-1083.

Schilling, S., Linker, R.A., Konig, F.B., Koziolek, M., Bahr, M., Muller, G.A., et al., 2006. Plasma exchange therapy for steroid-unresponsive multiple sclerosis relapses: clinical experience with 16 patients. Nervenarzt 77 (4), 430-438.

Sellebjerg, F., Barnes, D., Filippini, G., Midgard, R., Montalban, X., Rieckmann, P., et al., 2005. EFNS guideline on treatment of multiple sclerosis relapses: report of an EFNS task force on treatment of multiple sclerosis relapses. Eur. J. Neurol. 12 (12), 939-946.

Trebst, C., Reising, A., Kielstein, J.T., Hafer, C., Stangel, M., 2009. Plasma exchange therapy in steroid-unresponsive relapses in patients with multiple sclerosis. Blood Purif. 28 (2), 108-115.

Valbonesi, M., Garelli, S., Mosconi, L., Zerbi, D., Forlani, G., 1981. Plasma exchange in the management of patients with multiple sclerosis: preliminary observations. Vox Sang. $41(2), 68-73$.

Weiner, H.L., Dau, P.C., Khatri, B.O., Petajan, J.H., Birnbaum, G., McQuillen, M.P., et al., 1989. Double-blind study of true vs. sham plasma exchange in patients treated with immunosuppression for acute attacks of multiple sclerosis. Neurology 39 (9), 1143-1149.

Weinshenker, B.G., 1999. Therapeutic plasma exchange for acute inflammatory demyelinating syndromes of the central nervous system. J. Clin. Apher. 14 (3), 144-148.

Weinshenker, B.G., O'Brien, P.C., Petterson, T.M., Noseworthy, J.H., Lucchinetti, C.F., Dodick, D.W., et al., 1999. A randomized trial of plasma exchange in acute central nervous system inflammatory demyelinating disease. Ann. Neurol. 46 (6), 878-886. 